\title{
Thrombotic thrombocytopenic purpura with terminal pancytopenia
}

\author{
Soo-Chin $\mathrm{Ng}^{1}$ and B.A. Adam ${ }^{2}$ \\ ${ }^{1}$ Haematology Division, Department of Pathology, ${ }^{2}$ Department of Medicine, Medical Faculty, University \\ of Malaya, 59100 Kuala Lumpur, Malaysia
}

\begin{abstract}
Summary: A 27 year old housewife developed thrombotic thrombocytopenic purpura during the twelfth week of pregnancy. She had partial response to initial plasma infusion and subsequent plasmapheresis. However, her clinical course was complicated by the development of severe pancytopenia the consequence of a hypocellular marrow. She succumbed to septicaemic shock one month after diagnosis. The development of hypocellular marrow in thrombotic thrombocytopenic purpura has not been reported before.
\end{abstract}

\section{Introduction}

Thrombotic thrombocytopenic purpura (TTP) is a rare disorder characterized in its complete form by the pentad of thrombocytopenia, microangiopathic haemolytic anaemia (MAHA), fluctuating neurological abnormalities, renal impairment and fever. ${ }^{1}$ We report a patient with TTP in pregnancy who developed terminal pancytopenia secondary to hypocellular marrow.

\section{Case report}

A 27 year old housewife gravida 3, para 4 was admitted to a peripheral hospital with a history of fever for a week. She was 3 months pregnant. There were no localizing symptoms or signs and she gave no history suggestive of collagen diseases. Her general condition deteriorated rapidly and 4 days later she lapsed into a coma. Her haemoglobin concentration dropped from $10 \mathrm{~g} / \mathrm{dl}$ to $4 \mathrm{~g} / \mathrm{dl}$. She was transferred to the University Hospital, Kuala Lumpur for further management. On examination, she was in deep coma and responded only to deep pain. She was febrile with marked pallor and a tinge of jaundice. Her blood pressure was $120 / 80 \mathrm{mmHg}$. Both liver and spleen were enlarged $4 \mathrm{~cm}$ below the subcostal margins. Petechiae were noted over her lower limbs. No fundal haemorrhage or lymphadenopathy was detected. There were no focal neurological signs.

Her full blood count showed a haemoglobin concentration of $4.7 \mathrm{~g} / \mathrm{dl}$ with a reticulocyte count of $4 \%$. Her white cell count was elevated at

Correspondence: S.C. Ng, M.R.C.P. (UK), F.R.C.P.A. Accepted: 2 May 1990
$30.8 \times 10^{9} / 1$ with a slight left shift and the platelet count was $10 \times 10^{9} / 1$. The direct Coomb's test was negative. Peripheral blood film confirmed marked thrombocytopenia and a striking number of fragmented red cells and occasional nucleated red cells were present. Her prothrombin time, partial thromboplastin time, thrombin time and fibrinogen level were within normal limits. Bone marrow examination showed a normocellular marrow with erythroid hyperplasia and presence of normal granulopoiesis and megakaryopoiesis. Urinalysis revealed microscopic haematuria. Her blood urea was elevated $(18.9 \mathrm{mmol} / \mathrm{l})$ with serum creatinine of $115 \mu \mathrm{mol} / 1$. Her liver functions were essentially normal except for elevated serum bilirubin of $50 \mu \mathrm{mol} / \mathrm{l}$. Computerized axial scan of brain showed no focal lesions and cerebrospinal fluid (CSF) examination was normal. Cultures from blood, CSF and urine showed no growth. Ultrasound of abdomen revealed a viable fetus corresponding to 12 weeks' gestation. Serological tests for dengue and arbovirus and Widal Weil Felix tests were negative. The diagnosis of thrombotic thrombocytopenic purpura was made on the basis of microangiopathic haemolytic anaemia, profound thrombocytopenia, high fever, and evidence of both central nervous system and renal involvement.

She was started on plasma therapy consisting of daily infusion of 5 units of fresh frozen plasma (FFP). She made a remarkable recovery and regained full consciousness by the day 3 of admission. However, there was persistent MAHA and thrombocytopenia and she required 2 units of packed cell transfusions on alternate days. She was started on plasmapheresis on the day 5 of admission and subsequently six more plasmaphereses 
were performed over a period of 2 weeks (Figure 1). Each procedure involved an exchange of 2 litres of plasma with the replacement fluids consisting of 10 units of FFP and crystalloids. There was a partial response after each procedure - she became more alert and her serum creatinine and blood urea returned to normal. She also became afebrile on day 14 of admission but the MAHA and thrombocytopenia persisted. She had a spontaneous abortion on the same day. Her absolute neutrophil count declined markedly and was below $1 \times 10^{9} / 1$ on day 14. A bone marrow aspirate and trephine biopsy showed hypocellular marrow with depression of normal haemopoiesis. Three weeks after admissions, she became severely neutropenic $\left(<0.5 \times 10^{9} / 1\right)$; she was reverse isolated and started on broad spectrum antibiotics but became febrile again and was deeply jaundiced on day 22 . Staphylococcus aureus was isolated in the blood culture. Her coagulation tests showed evidence of disseminated intravascular coagulation. Alanine transaminase (ALT) was not raised at any time. Despite vigorous treatment, she succumbed to septicaemic shock 30 days after admission.

\section{Discussion}

TTP was first described by Moschcowitz in $1925 .{ }^{2}$ In more than $90 \%$ of patients, TTP developed without an apparent causal event or an underlying disease process. ${ }^{3}$ In a minority of cases, the disorder has been associated with a variety of conditions including collagen diseases, antecedent respiratory infections, drugs and pregnancy. Despite a relatively high incidence in women of child bearing age, TTP has rarely been reported during pregnancy. 4,5 Our patient was 12 weeks pregnant at presentation of illness but there is no convincing evidence that the above associations are causal rather than coincidental. ${ }^{6}$

Although the aetiology and pathogenesis of the condition remain unknown, the introduction of therapy designed to remove the patient's plasma and/or to supply the patient with normal plasma has dramatically improved the prognosis of TTP. Up to $70-80 \%$ response rate has been reported for TTP patients treated with plasma infusions, ex-

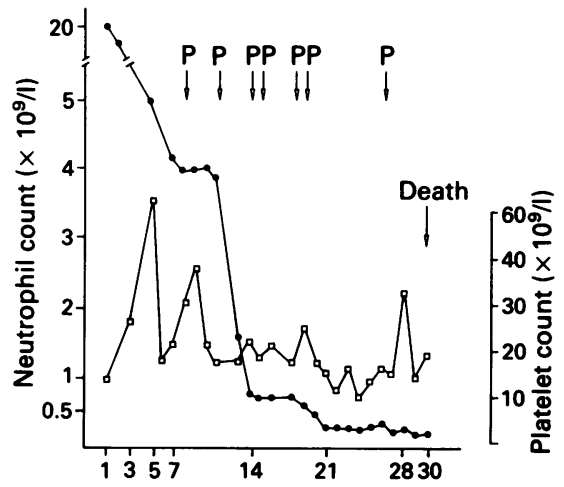

Figure 1 Clinical course of patient. count; $\square-\square$, platelet count; P, plasmapheresis.

change transfusions or plasmapheresis. ${ }^{7,8}$ Our patient responded partially to plasma infusions and plasmapheresis but unfortunately her clinical course was complicated by the development of severe pancytopenia, the consequence of a hypocellular marrow. Hypoplastic/aplastic anaemia has rarely been reported in pregnancy. ${ }^{9}$ The precise relationship between pregnancy and aplasia remains uncertain but it is likely to be a chance association. ${ }^{10}$ The cause of the hypocellular marrow in our patient was not apparent. Non-A, non-B hepatitis causing hypocellular marrow was not likely in view of her normal ALT. Transfusion associated graft-versus-host disease could follow blood product transfusions in patients with deficient cell-mediated immunity. ${ }^{11}$ However, the absence of skin lesions and gastrointestinal complaints and her normal ALT made it unlikely that her bone marrow failure was due to graft-versus-host disease. Moreover, there was no suggestion that the patient had deficient cell-mediated immunity. One may speculate that the same aetiological agent that triggered off TTP also caused the hypocellular marrow.

\section{Acknowledgements}

We thank Dr M.L. Ong and Dr Keith Lim for helping with patient care.

\section{References}

1. Amorosi, E.L. \& Ultmann, J.E. Thrombotic thrombocytopenic purpura: report of 16 cases and review of the literature. Medicine 1966, 45: 139-159.

2. Moschcowitz, E. An acute febrile pleiochromic anaemia with hyaline thrombosis of the terminal arterioles and capillaries. Ann Intern Med 1925, 31: 89-93.
3. Ridolf, R.L. \& Bell, W.R. Thrombotic thrombocytopenic purpura: report of 25 cases and review of the literature. Medicine 1981, 60: 413-428.

4. Yang, C., Nussbaum, M. \& Park, H. Thrombotic thrombocytopenic purpura in early pregnancy. Acta Haematol 1979, 62: 112-116. 
5. Byrnes, J.J. \& Khurana, M. Treatment of thrombotic thrombocytopenic purpura with plasma. N Engl J Med 1977, 297: 1386-1389.

6. Crain, S. \& Choudhury, A. Thrombotic thrombocytopenic purpura, a reappraisal. JAMA 1981, 246: 1243-1246.

7. Cutter, J. Thrombotic thrombocytopenic purpura: A ten year experience. Blood 1980, 56: 302-306.

8. Blitzer, J.B., Granfortuna, J.M., Gottileb, A.J. et al. Thrombotic thrombocytopenic purpura: treatment with plasmapheresis. Am J Hematol 1987, 24: 329-339.
9. Fleming, A.F. Hypoplastic anaemia in pregnancy. Clin Haematol 1973, 2: 477-496.

10. Blanche, P.A., Nancy, U.P. \& Frederick, P.L. Classification and aetiology of the aplastic anaemias. Clin Haematol 1978. 7: $431-457$.

11. Brubaker, D.B. Human posttransfusion graft-versus-host disease. Vox Sang 1983, 45: 401-420. 